\title{
Movilidad residencial y social en barrios populares consolidados en Bogotá
}

\author{
Mobilidade residencial e social em bairros populares \\ consolidados em Bogotá
}

\author{
Residential and social mobility in consolidated popular \\ neighborhoods in Bogotá
}

Angélica Camargo Sierra [a] [D, Alex Smith Araque Solano [b] (iD)

\author{
[a] Universidad Externado de Colombia, Bogotá, Colombia \\ [b] Universidad Sergio Arboleda, Escuela de Economía, Bogotá, Colombia
}

Cómo citar: Camargo, A., \& Araque, A. (2021). Movilidad residencial y social en barrios populares consolidados en Bogotá. urbe. Revista Brasileira de Gestão Urbana, v.13, e20200109. https://doi.org/10.1590/2175-

3369.013.e20200109

\section{Resumen}

En este texto se estudia la movilidad social y residencial de los propietarios de viviendas en algunos barrios populares consolidados en Bogotá. La trayectoria laboral y residencial del propietario antes de su llegada a la vivienda actual permite acercarse a la movilidad social ocupacional intrageneracional. Mediante el enfoque biográfico se analizaron 341 encuestas a propietarios en cinco barrios populares consolidados. Los datos permiten concluir que tienen trayectorias estáticas, tanto a nivel ocupacional como residencial. Las razones explicativas de la inmovilidad ocupacional y residencial se encuentran en aspectos estructurales: bajo nivel educativo, reducidos cambios de trabajo y bajos ingresos. Construir una vivienda grande en un largo periodo de tiempo y albergar varios hogares con vínculo familiar inmoviliza a los propietarios ocupacional y residencialmente, excepto que logren movilizar la vivienda como activo mediante el alquiler de espacios. Se discute sobre el papel de la propiedad de la vivienda en la confluencia de estas inmovilidades y se reflexiona sobre la reproducción de las desigualdades en el marco de la política habitacional.

Palabras clave: Movilidad residencial. Movilidad social. Barrios populares. Política habitacional.

\section{Resumo}

O objetivo do texto é analisar a mobilidade social e residencial de proprietários localizados em bairros populares consolidados de Bogotá. A mobilidade ocupacional social intrageneracional foi analisada através da leitura da trajetória de trabalho, e a trajetória residencial do proprietário também foi reconstruída antes de sua chegada à atual residência. Utilizando a abordagem biográfica, 341 inquéritos aos proprietários foram analisados em cinco bairros populares consolidados. Os dados permitem concluir que eles têm trajetórias estáticas, tanto no

ACS es arquitecta, investigadora, doctora en Estudios Sociales, e-mail: angelicapcamargos@yahoo.com ASAS es magister en Economía, e mail: alex.araque@usa.edu.co 
nível ocupacional quanto no nível residencial. As razões explicativas da imobilidade ocupacional e residencial são encontradas em aspectos estruturais: baixa escolaridade, redução de trocas de emprego e baixa renda. Construir uma casa grande por um longo período e abrigar várias casas ligadas à família imobiliza os proprietários ocupacional e residencialmente, exceto que eles conseguem mobilizar a casa como um ativo alugando espaço. $O$ papel da propriedade da casa na confluência dessas imobilizações é discutido e é feita uma reflexão sobre a reprodução das desigualdades no âmbito da política habitacional.

Palavras-chave: Mobilidade residencial. Mobilidade social. Bairros populares. Política habitacional.

\section{Abstract}

The main goal of this paper is to analyze the social and residential mobility of homeowners in consolidated neighborhoods in Bogotá. The occupational social mobility was analyzed at an intragenerational level by reading their job trajectory, and the owner's residential trajectory was reconstructed before arriving at the current house. With the biographical approach, 341 surveys of owners were conducted in five consolidated neighborhoods in Bogotá. The data lead to the conclusion that the owner's trajectories are very static, both from the occupational point of view and residential. The reasons for the occupational and residential immobility were found in structural aspects: the low educational level and the small number of job changes added to a low income. Building a large house over a long period and share the house with several households with familiar link ties immobilizes the owners both at occupational and residential levels, except when the owner mobilizes their house as an asset by renting spaces. Finally, we discuss the role of homeownership in the confluency of the social and residential immobility and the reproduction of inequality.

Keywords: Residential mobility. Social mobility. Popular neighborhoods. Housing policy.

\section{Introducción}

Cesar vive en San Martín de Porres en Chapinero, cerca de los barrios de alta renta de Bogotá, a escasos kilómetros del centro de la ciudad, sobre los cerros orientales, con una gran vista de la ciudad a pocos metros de las principales Universidades de Bogotá. Su esposa, Susana, heredó la vivienda en donde han vivido toda su vida. Al unirse empezaron la construcción de su vivienda después de demoler la casa de sus padres. Cesar ha trabajado siempre como vendedor ambulante; sus ingresos laborales han sido estables en el largo plazo y tienden a disminuir recientemente. No obstante, en su vivienda autoconstruida recibe el alquiler de cinco apartamentos de dos alcobas, nueve de una alcoba y un local. La trayectoria ocupacional de Cesar es estática y su movilidad residencial inexistente. Sin embargo, es posible entrever un proceso de movilidad social ascendente a nivel intra e intergeneracional. Los cerca de 2500 dólares mensuales percibidos por arriendos le han permitido adquirir otros bienes y educar a sus dos hijas como profesionales. Aunque ha vivido siempre en la misma vivienda, su entorno actual es diferente al de hace cuarenta años; su localización relativa es muy superior. Su caso evidencia la complejidad de las trayectorias de vida de los hogares en los sectores populares, particularmente de las articulaciones entre la movilidad social y residencial de estas familias, y sobretodo el papel de la vivienda en los procesos de ascenso social, que constituye el tema central de esta investigación. ¿Se pueden evidenciar procesos de movilidad social ascendente (o descendente) en las carreras laborales de los propietarios? ¿Qué aspectos socioeconómicos y residenciales han determinado tales procesos de movilidad social? ¿Cómo se configuran y qué aspectos explican las trayectorias residenciales que dieron lugar a la posición actual de los propietarios? ¿Qué articulaciones o relaciones hay entre las trayectorias laborales y residenciales de los propietarios de estos barrios? Son las preguntas que guían las reflexiones de este artículo.

Este estudio se articula con una red de investigación interesada por estudiar aquellos asentamientos de origen irregular localizados en las primeras periferias urbanas de las ciudades de América Latina y que surgieron hace más de 30 años. Se analizarán, en particular, los hogares de los propietarios de viviendas localizadas en barrios populares consolidados en Bogotá que surgieron entre 1950 y 1985. 
Mediante el uso de un enfoque biográfico, se indagó la trayectoria residencial de los propietarios referida al conjunto de cambios de residencia a lo largo de la vida (Authier et al., 2010). Además, se rastró la trayectoria ocupacional correspondiente a cada una de las posiciones laborales mayores a un año ocupadas por el encuestado. Se realizaron 341 encuestas en cinco barrios populares de Bogotá: San Martín de Porres, Atenas, El Anhelo, Japón e Ismael Perdomo ${ }^{1}$ (Ver Mapa 1). Estos barrios se encuentran actualmente integrados a la ciudad y por tanto se califican como "consolidados". Si bien cuando se formaron estaban ubicados en zonas periféricas, hoy en día, debido al proceso de crecimiento urbano, están ubicados en áreas relativamente cercanas al centro urbano, tienen todos los servicios públicos y urbanos, buena accesibilidad al resto de la ciudad, vías pavimentadas y diferentes rutas y alternativas de transporte público.

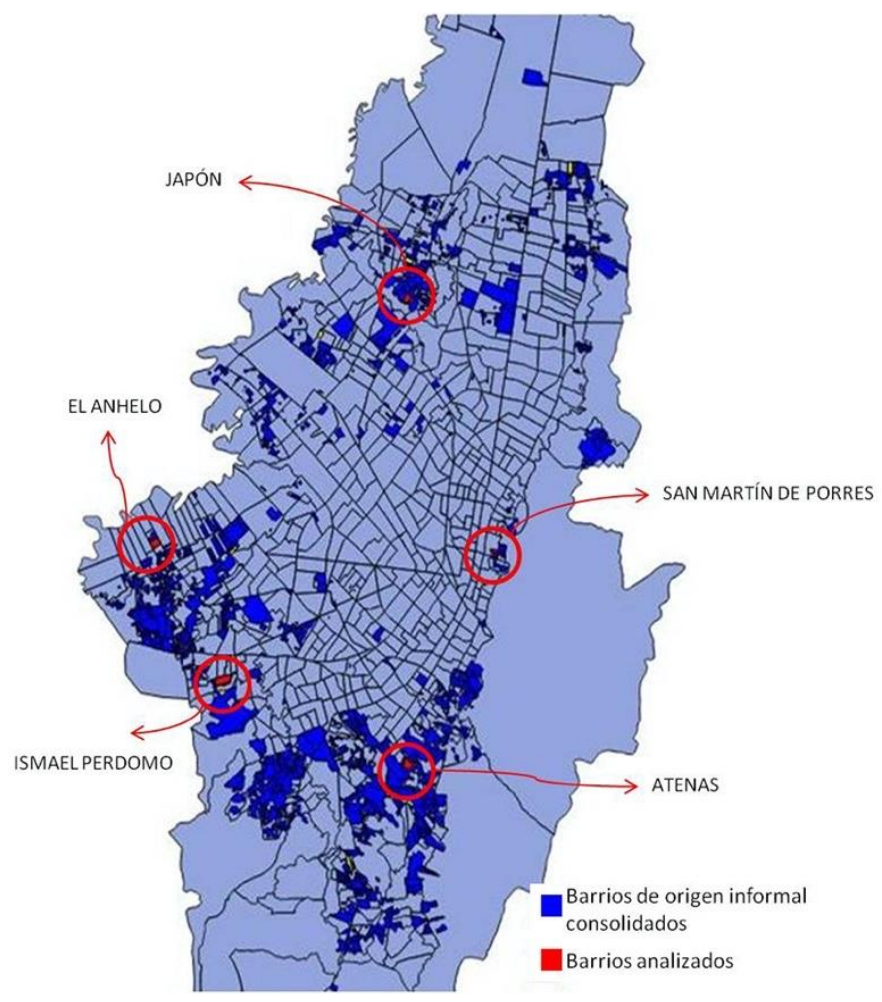

Mapa 1 - Localización de los barrios analizados. Fuente: Elaboración propia con datos de la Secretaría Distrital de Planeación de Bogotá (2018).

En la primera parte del texto se presentan algunas reflexiones conceptuales sobre la movilidad residencial y social. Posteriormente, se presentan los principales hallazgos del estudio, se estimaron acá un conjunto de modelos econométricos que permitieran explicar aspectos de las trayectorias los propietarios. Finalmente, se concluye con algunas reflexiones orientadas a las políticas públicas habitacionales y urbanas.

\section{La movilidad residencial en los sectores populares}

La movilidad residencial se define como los cambios residenciales a nivel intraurbano (Dieleman, 2001). Para Knox (1982), es un producto entre las oportunidades (a nivel de mercado inmobiliario, ingresos

\footnotetext{
1 Se realizó un muestreo representativo de los asentamientos de origen informal consolidados en Bogotá que reportaran un surgimiento entre 1955 y 1985. Se escogieron 5 barrios de manera sistemática, mediante un análisis multicriterio ponderado con la totalidad de los barrios, se utilizó así una estrategia de múltiples casos que en su conjunto pudieran dar cuenta del universo de estudio (Stake, 1994). Se realizó una muestra probabilística con tamaño máximo, dado un nivel de confianza del 95\%, se determinó un porcentaje de error correspondiente al $5 \%$ arrojando un tamaño de muestra de 388 encuestas 341 respondidas por propietarios y 47 por arrendatarios). La unidad de muestreo fue el lote en donde había varios predios. La elección de los lotes se realizó de manera aleatoria. La entrevista se dirigía al propietario de la casa, en segundo lugar, su cónyuge y en tercero un hijo mayor de edad, cuando se reportó que el propietario no vivía en la casa se entrevistó un arrendatario (47 casos). Las encuestas se realizaron entre marzo y abril de 2015.
} 
y posibilidades de los hogares) y las necesidades y expectativas residenciales de las familias. Se trata de un proceso complejo en el que se interrelacionan múltiples elementos. A nivel del hogar o del individuo hay factores que determinan las decisiones de moverse o no de residencia tales como la posición en la estructura social, los ingresos, la composición del hogar, el ciclo de vida, las preferencias, el conocimiento sobre el mercado inmobiliario, entre otros. A nivel macro, el funcionamiento de los mercados laboral e inmobiliario, las políticas habitacionales, la desigualdad y el patrón de segregación urbana, entre otros, determinan las dinámicas de movilidad de los individuos y hogares. Asimismo, los procesos de movilidad residencial, configuran parte de estos factores estructurales, hay así una interacción entre las dinámicas de movilidad residencial y el comportamiento de los factores estructurales mencionados, lo que Knox denomina "la dialéctica socioespacial" (p. 252).

Estudio pioneros como los de Rossi (1955) y Brown \& Moore (1970), han analizado las determinantes de las decisiones y elecciones residenciales. Otros estudios en los años 90, analizaron las determinantes de las decisiones residenciales y la dinámica del mercado de viviendas en relación con la movilidad residencial (Dieleman, 2001). En Francia, según Authier et al., (2010), se han abordado dos aspectos centrales, la noción de estrategia y la importancia de la familia en el análisis de las trayectorias residenciales. En España, se encuentran trabajos como los de Módenes (2008), Pujadas (2009) y Leal (2002). Se han realizado mediciones y modelos explicativos de las dinámicas de inmovilidad residencial como los de Pajares Linares \& Van Ham (2017).

En América Latina el estudio de estos temas es relativamente reciente ${ }^{2}$. La mayoría han caracterizado los procesos y dinámicas de movilidad residencial en términos de su intensidad, preferencias localizacionales, cambios en el estatus de propiedad y tipos de vivienda en diferentes zonas metropolitanas y en diferentes categorías socioeconómicas, en algunos casos en relación con procesos de segregación.

En cuanto al análisis de los procesos de movilidad residencial en los sectores populares, Dureau et al. (2015), señalan dinámicas contrastantes en la intensidad de la movilidad residencial. Los arrendatarios que habitan en sectores populares son los más móviles mientras los propietarios los más inmóviles. De acuerdo con Gilbert (2001) la inmovilidad de los propietarios genera problemas en la calidad de vida de las familias. Abramo (2008) encuentra, sin embargo, un mercado de compraventa de viviendas dinámico en las favelas de Brasil, incluso más que el mercado formal. Álvarez (2007) en Montevideo, señala también una alta movilidad residencial en barrios informales en esta ciudad.

Estudios longitudinales como los de Gilbert (2001) y Ward (2009) encontraron en Bogotá y México respectivamente, que en los barrios que visitaron treinta años antes, el $80 \%$ de los propietarios iniciales todavía seguía en su vivienda. No obstante, Perlman (2010) con una metodología similar encontró, en Rio de Janeiro, que solo 37\% de los propietarios originales permanecía en la Favela. Según Padilla (2012), en Guadalajara, si bien los padres no se han movido de la vivienda en más de 20 años, los hijos salen y regresan a la vivienda según cambian sus condiciones laborales o maritales.

\section{Movilidad social y su relación con aspectos residenciales}

El concepto de movilidad social refiere a los cambios en las condiciones socioeconómicas de los individuos o familias a través del tiempo y su proyección en la posición en la estructura social jerárquica. Según Sorokin (1961) se refiere a "toda transición de un individuo, objeto o valor social de una posición social a otra" (p. 135). La movilidad social puede ser ascendente, estable o descendente, y su estudio analiza los factores que la promueven sus canales o determinantes (Uribe \& Pardo, 2006).

La movilidad social solo es posible cuando la sociedad está estructurada en clases sociales, es decir con algún nivel de desigualdad. En este marco, se asocia con la igualdad de oportunidades, y se pregunta ¿qué tan abiertas son las oportunidades de progreso en una sociedad? (Kessler \& Espinoza, 2003). En una

2 En Chile: Contreras (2012) y Rodríguez (2007). En Brasil Menna (2000), y Abramo (2008). En Argentina Di Virgilio (2011) y en México investigaciones de Duhau (2003), Zamorano (2007) y Suárez \& Delgado (2010). En Colombia: Lulle (1998) y Dureau (2010). 
perspectiva temporal (intra e intergeneracional) indaga ¿qué tanto los individuos y sus herederos permanecen en la misma ubicación social? De tal modo, "un sistema altamente desigual concentra ventajas en la cima de su jerarquía y fija a los individuos o unidades sociales en sus posiciones dentro de ella" (Tilly, 2008, p. 53).

El análisis de la movilidad social tiene un punto de origen y un destino, con dos posibilidades analíticas, intra e intergeneracional. En la perspectiva intergeneracional el punto de origen es la clase social de los padres y el destino la de los hijos adultos (Torche \& Wormald, 2004). Desde la perspectiva intrageneracional origen y destino se ubican en puntos del tiempo en la vida de las personas. En este texto se abordará una perspectiva intrageneracional en la mirada a la movilidad social ocupacional de los propietarios.

Algunos estudios han explorado la relación entre la movilidad social y determinados aspectos residenciales. Valenzuela \& Herrera (2003), analizan en Chile los cambios de residencia entre 1992 y 2002, para establecer la relación entre la movilidad residencial y social. Observan si los cambios de residencia se producen hacia zonas con mejor acceso a bienes y servicios respecto a las zonas de origen. Concluyen que las movilidades tienden a ser ascendentes.

En Bogotá Lulle (1998), adelantó un estudio para las clases medias con el objetivo de "identificar las prácticas residenciales de los estratos medios bogotanos y evidenciar los efectos de la movilidad laboral y social sobre ellas" (p. 467). Encuentra una simultaneidad temporal entre la movilidad residencial y social. Uribe \& Pardo (2006), en Bogotá, identifican la movilidad residencial como una expresión de la movilidad social. Zamorano (2007) en México, encuentra que no siempre una movilidad residencial es resultado o consecuencia de un proceso de movilidad social.

El acceso a la propiedad ha sido tradicionalmente entendido como una forma de movilidad social ascendente. Para los hogares, la propiedad se percibe como un ascenso social (Bonvalet \& Dureau, 2000; Lulle, 1998). Ciertamente, la propiedad de la vivienda permite mayor seguridad de la tenencia y promueve la generación de ingresos adicionales. Además, representa un soporte importante para la vejez y la seguridad económica de la segunda e incluso tercera generación. (Bonvalet \& Dureau, 2000; Di Virgilio, 2011; Gilbert, 2001). Sin embargo, los propietarios de bajos ingresos son quienes reportan mayor inmovilidad residencial (Dureau, 2010; Delaunay \& Dureau, 2004; Uribe \& Pardo, 2006). Así, en contraste con las ventajas de la propiedad ya mencionadas, la inmovilidad residencial de los propietarios de bajos recursos, encierra desventajas relacionadas con restricciones para cambiar cuando las necesidades o aspiraciones cambian (Gilbert, 2001, p. 73).

\section{Características de las viviendas y los hogares}

En la tabla No 1 se presentan los estadísticos que evalúan la tendencia central en las áreas de terreno y de construcción y su discriminación por barrios. En la primera columna se tiene el barrio, luego los valores máximos (Max), la media y el coeficiente de variación (CV). El área promedio de terreno se acerca a 120 metros cuadrados. El coeficiente de variación indica que la desviación estándar es el 63\% de la media de los terrenos.

Tabla 1 - Características de las viviendas y los hogares

\begin{tabular}{lllllll}
\hline & Área del Terreno & \multicolumn{3}{l}{ Área Construida } \\
\hline Barrio/Estadístico* & Media & Max & CV & Media & Max & CV \\
Total & 119 & 374 & 0,63 & 156,2 & 420 & 0,49 \\
Atenas & 118 & 360 & 0,52 & 164 & 280 & 0,41 \\
El Anhelo & 67 & 81 & 0,23 & 123 & 270 & 0,44 \\
Japón & 84,3 & 192 & 0,55 & 182 & 270 & 0,29 \\
Perdomo & 180 & 340 & 0,43 & 219 & 345 & 0,36 \\
San Martín de Porres & 109 & 374 & 0,72 & 158 & 420 & 0,6 \\
\hline
\end{tabular}

Fuente: Cálculos de los autores. 
Las cifras muestran importantes diferencias entre barrios. En el Anhelo, San Martín de Porres y Japón tienen los lotes tienen una moda en 72 metros cuadrados. En el Perdomo los predios son más grandes, con una moda de 250 y otra en 125 metros cuadrados, su mediana es inferior a la media y su coeficiente de variación es bajo comparado con el total, la desviación estándar es el 43\% de la media. Si bien el área de los lotes es bastante heterogéneo, se aprecia una concentración de lotes menores de 84 metros cuadrados.

Respecto de las áreas construidas, los propietarios calculan tener construidos 156 metros cuadrados en promedio. Las edificaciones no son muy altas, la mayoría tiene uno (22\%), dos (50\%) o tres pisos (24\%), solo en Japón y San Martín de Porres las edificaciones superan los dos pisos en el 50\% de los registros. Si bien cada edificación corresponde a un solo predio (catastral) y generalmente un solo propietario, las casas suelen estar subdivididas. El 64,82\% de los propietarios tiene por lo menos 2 hogares por edificación.

La forma de subdivisión común es por pisos, suele construirse en cada piso uno o dos apartamentos independientes. Las casas tienen en promedio cinco alcobas, una sala y/o comedor, dos cocinas, dos baños y un patio o terraza. El 32,5\% no tiene patio, la mayoría (70\%) no tiene garaje y el 31,6\% tiene local. En síntesis, las edificaciones son relativamente grandes y suelen estar subdividas en apartamentos por piso. Cada apartamento cuenta con alcobas, cocina y baño, la mayoría (89\%) tiene entrada independiente y en algunos casos los servicios independizados (en el 38,5\% de los casos). En cada edificación viven en promedio 2,2 hogares y 7,5 personas. El tamaño de los hogares (4,0 personas por hogar) de los propietarios de los barrios escogidos es en general más grande que el promedio de la ciudad ${ }^{3}$ (Ver tabla 2).

Tabla 2 - Tipología de los hogares

\begin{tabular}{lll}
\hline Tipo & Porcentaje & Personas/hogar \\
\hline Unipersonal & $5,2 \%$ & 1,0 \\
Nuclear & $56,9 \%$ & 3,5 \\
Extendido & $35,2 \%$ & 5,2 \\
Compuesto & $0,9 \%$ & 3,6 \\
No nuclear & $0,1 \%$ & 3,7 \\
\hline
\end{tabular}

Fuente: Elaboración propia con datos de la encuesta a propietarios

El porcentaje de hogares extensos es alto con referencia al de la ciudad ${ }^{4}$ que apenas sobrepasa el $20 \%$ y el tamaño promedio de los hogares se ve influenciado por el tamaño de los hogares extensos (Tabla 2). Las diferencias de edades promedio entre padres $(59,4)$, hijos $(25,9)$ y nietos $(12,2)$ indican que los hogares están en las últimas etapas de su ciclo familiar que es cuando los hijos conforman sus propios hogares y dejan el hogar paterno.

\section{Trayectorias laborales de los propietarios, una mirada a la movilidad social ocupacional intrageneracional}

El análisis de las ocupaciones permite analizar la movilidad social en virtud de la centralidad del trabajo como fundamento de la vida social y del acceso a las oportunidades sociales Torche \& Wormald (2004, p. 10). Una de las clasificaciones comúnmente usadas en los estudios sobre movilidad social es la de Erikson \& Goldthorpe (1992), que en un ejercicio de adaptación a la sociedad latinoamericana Torche \& Wormald (2004) agruparon en ocho clases. Para este trabajo se optó por utilizar el nivel de cualificación de las ocupaciones $^{5}$ establecido en la Clasificación Nacional de Ocupaciones del 2015. Luego, se agruparon las

\footnotetext{
3 En Bogotá, para 2011, el promedio de personas por hogar fue de 3,4 y para 2014 éste se redujo a 3,2 personas por hogar.

4 21,5\% según la encuesta multipropósito de Bogotá 2014 (Secretaría Distrital de Planeación, 2015).

5 Definido "por la complejidad de las funciones, el nivel de autonomía y responsabilidad en el desempeño de la ocupación en relación con otras y, por consiguiente, la cantidad, tipo y nivel de educación, capacitación y experiencia requeridos para su desempeño" (SENA \& Ministerio del Trabajo, 2015, p. 10).
} 
principales ocupaciones encontradas y se homologaron con a las categorías de Torche \& Worldman, tal como aparece en la Tabla 3.

Tabla 3 - Ocupaciones identificadas en la encuesta según clasificación y nivel ocupacional y categorías Torche y Worldman

\begin{tabular}{|c|c|c|}
\hline $\begin{array}{l}\text { Clasificación nacional de ocupaciones } \\
2015\end{array}$ & Torche y Worldman & $\begin{array}{l}\text { Ocupaciones identificadas en las } \\
\text { trayectorias }\end{array}$ \\
\hline $\begin{array}{l}\text { A: Funciones variadas y complejas, alto } \\
\text { grado de autonomía, responsabilidad } \\
\text { estudios universitarios o a nivel de postgrado. }\end{array}$ & $\begin{array}{l}\text { I clase de servicios } \\
\text { Il clase de rutina no } \\
\text { manual }\end{array}$ & $\begin{array}{l}\text { Trabajos profesionales, profesores de } \\
\text { colegio y universidad. }\end{array}$ \\
\hline $\begin{array}{l}\text { B: Funciones muy variadas, } \\
\text { responsabilidad de supervisión, estudios } \\
\text { técnicos o tecnológicos. }\end{array}$ & $\begin{array}{l}\text { III pequeña burguesía } \\
\text { IV trabajadores } \\
\text { independientes }\end{array}$ & $\begin{array}{l}\text { Administrador de un negocio propio, } \\
\text { trabajo técnico (topógrafo, auxiliar de } \\
\text { enfermería), asistente administrativo y } \\
\text { asesor comercial. Trabajadores } \\
\text { independientes calificados. }\end{array}$ \\
\hline $\begin{array}{l}\text { C: Funciones que combinan actividades } \\
\text { físicas e intelectuales, programa de } \\
\text { aprendizaje para el trabajo, educación } \\
\text { básica secundaria más cursos de } \\
\text { capacitación, entrenamiento en el trabajo } \\
\text { o experiencia. }\end{array}$ & $\begin{array}{l}\text { V Trabajadores manuales } \\
\text { calificados }\end{array}$ & $\begin{array}{l}\text { Modistería, carpintería, obrero de } \\
\text { construcción, cuidado infantil, estética, } \\
\text { policía, soldado, mecánico de carros, } \\
\text { panadero, secretaria, recepcionista, } \\
\text { operario, vigilante o conductor. }\end{array}$ \\
\hline $\begin{array}{l}\text { D: Funciones sencillas y repetitivas, } \\
\text { fundamentalmente de carácter físico, } \\
\text { con alto nivel de subordinación; la } \\
\text { experiencia laboral requerida es mínima, } \\
\text { educación básica primaria. }\end{array}$ & $\begin{array}{l}\text { VI Trabaja manuales no } \\
\text { calificados } \\
\text { VI Pequeños propietarios } \\
\text { agrícolas } \\
\text { VII Trabajadores agrícolas }\end{array}$ & $\begin{array}{l}\text { Agricultor, servicios generales (servicio } \\
\text { doméstico, ayudante de cocina, } \\
\text { aseador-a, auxiliares de } \\
\text { mantenimiento, auxiliar de ruta, lavado } \\
\text { de ropa) vendedor ambulante. }\end{array}$ \\
\hline
\end{tabular}

Fuente: Elaboración propia con datos recogidos en la encuesta y con base en la Clasificación Nacional de Ocupaciones 2015, SENA \& Ministerio del Trabajo (2015), y Torche \& Worldman (2004).

Inicialmente, se cuantificaron los cambios de trabajo mayores a un año registrados en la trayectoria laboral de los encuestados. Los propietarios han tenido en promedio 2.2 trabajos. La edad promedio de inicio de la vida laboral es de 17 años. En solo el 4.5\% (13 casos) el propietario entrevistado no ha trabajado nunca. El 30.4\% (75 casos) son poco móviles laboralmente (no han cambiado nunca de trabajo o no han trabajado). La mayoría ha tenido entre 2 y 3 trabajos, y el $11.3 \%$ ha tenido 4 o más. Para analizar la movilidad social ocupacional se procesaron los datos de la primera y la última categoría ocupacional alcanzada por los propietarios en una matriz de origen destino con las cuatro categorías de la tabla 3. La zona sin color de la Tabla 4 corresponde a quienes descendieron de categoría ocupacional, la gris los que se mantuvieron la categoría y la oscura aquellos que ascendieron.

Tabla 4 - Matriz origen destino movilidad social ocupacional de los propietarios a nivel intrageneracional

\begin{tabular}{|c|c|c|c|c|c|c|c|c|}
\hline \multirow{3}{*}{$\begin{array}{l}\text { Categoría } \\
\text { Primer trabajo }\end{array}$} & \multicolumn{8}{|c|}{ Categoría última o actual ocupación } \\
\hline & A & & B & & C & & D & \\
\hline & $\mathbf{N}$ & $\%$ & $\mathbf{N}$ & $\%$ & $\mathbf{N}$ & $\%$ & $\mathbf{N}$ & $\%$ \\
\hline A & 2 & $0,8 \%$ & 0 & $0,0 \%$ & 0 & $0,0 \%$ & 0 & $0,0 \%$ \\
\hline B & 0 & $0,0 \%$ & 1 & $0,4 \%$ & 1 & $0,4 \%$ & 0 & $0,0 \%$ \\
\hline C & 1 & $0,4 \%$ & 6 & $2,4 \%$ & 61 & $29,2 \%$ & 19 & $8,5 \%$ \\
\hline D & 2 & $0,6 \%$ & 5 & $2,1 \%$ & 32 & $13,5 \%$ & 89 & $41,8 \%$ \\
\hline
\end{tabular}

Fuente: elaboración propia con datos de encuesta a propietarios.

El 72.2\% se mantuvo la categoría ocupacional durante toda su trayectoria. El $18 \%$ tuvo movilidad ascendente, aunque en general de corto alcance, pasando de trabajos manuales no calificados a trabajos manuales calificados. En solo 11 casos ascendieron a categoría B y en solo tres a categoría A. El 8.9\% tuvo una movilidad social laboral descendente, también de corto alcance, casi todos de categoría $\mathrm{C}$ a $\mathrm{D}$, y en solo 
un caso de categoría B a C. Las trayectorias laborales de los propietarios han sido estables. La mayoría se mantuvo en la misma categoría ocupacional a largo de su trayectoria. En los pocos casos en que experimentaron una movilidad social laboral, fue de corto alcance.

\section{Inmovilidad social ocupacional y los aspectos residenciales}

Para aproximarse a los aspectos que configuran la estabilidad social ocupacional en estos grupos de población se explora su relación con variables asociadas al individuo como el número de cambios de trabajo y el nivel educativo (primaria, secundaria, tecnológica y profesional). Variables asociadas a la vivienda y el hogar como el número de metros cuadrados construidos de la vivienda (lo que sugiere un gran esfuerzo generacional), los ingresos del hogar que se clasificaron por rangos, la cantidad de hogares por vivienda, el número de hogares familiares del propietario en la misma edificación. Se incluyeron variables de percepción como los aspectos que considera importantes para mejorar económicamente y el nivel de satisfacción con la vida. Se utilizó un modelo probit en donde el trabajador inmóvil toma el valor de uno y cero si asciende o desciende, como variable explicada (ver tabla 5).

Tabla 5 - Resultados modelo inmovilidad laboral

\begin{tabular}{lll}
\hline Ingresos & Estimador & $\mathbf{z}$ \\
\hline Cambios de trabajo &,- 972453 & $-2,12$ \\
Área edificada &, 0302024 & 2,02 \\
Hogares por vivienda & $-1,740678$ & $-1,92$ \\
Red familiar & 1,985036 & 2,11 \\
Nivel educativo & & \\
$\quad$ Primaria & $-2,595118$ & $-1,21$ \\
$\quad$ Secundaria & 2,322383 & 1,43 \\
$\quad$ Técnico/tecnológico & $-2,619324$ & $-1,08$ \\
$\quad$ Entre 300.000 y 600.000 & & 2,34 \\
$\quad$ Entre 601.000 y 1.200.000 & 5,411495 & 1,50 \\
$\quad$ Entre 1.200.001 y 2.400.000 & 2,012013 & 1,45 \\
$\quad$ Trabajar muy duro & 2,059112 & 0,31 \\
Tener un empleo formal y estable & $-4,205037$ & $-2,15$ \\
Tener una casa grande y bien ubicada & 1,330627 & 0,70 \\
Tener fe en dios & $-1,755648$ & $-1,67$ \\
Aspectos para mejorar en la vida & & $-0,51$ \\
Bastante satisfecho &,- 4422418 & 1,42 \\
Solo satisfecho & 3,165493 & \\
Pseudo R2 &, 7680972 & 0,5192 \\
\hline
\end{tabular}

Fuente: Cálculos de los autores.

En primer lugar, el modelo tiene un nivel adecuado de clasificación. De cada 100 individuos, el modelo estima correctamente 85 dentro del grupo de estabilidad ocupacional. En segundo lugar, los resultados de la tabla 5 solo dicen significancia estadística y adquieren sentido con la figura 1 donde se presentan los cambios de probabilidad. En la primera columna se encuentran las variables, en la segunda el estimador y en la tercera el estadístico de prueba de la irrelevancia estadística de la variable. En valor absoluto, todo z $>2$ rechaza que el estimador es cero. Las variables factores solo se describen, no son ilustrativas de los cambios de probabilidad. 
Los resultados sugieren que, en estos grupos poblacionales, los individuos que cambian de trabajo reducen la probabilidad de estabilidad social ocupacional. El análisis de las probabilidades de la Figura 1 muestra que la probabilidad de ser inmóvil ocupacional con 0 cambios de trabajo es del $97 \%$. Con un cambio de trabajo se tiene una probabilidad del 93\% ser inmóvil mientras que con 3 cambios de trabajo la probabilidad se reduce al 70\%. Podría considerarse una reducción marginal pero dada la precariedad del capital humano es una variable que considerar para la política al involucrar la educación.

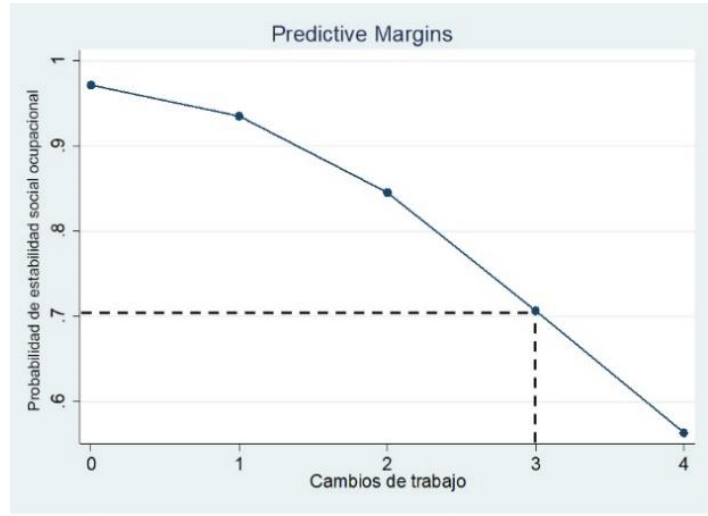

(a)

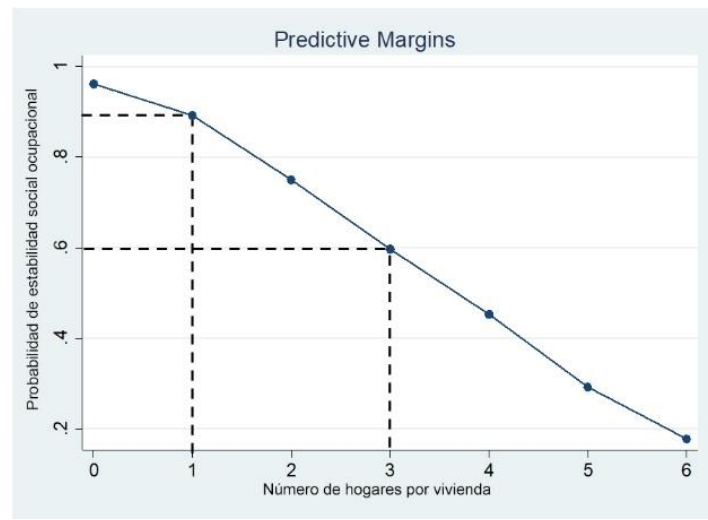

(c)

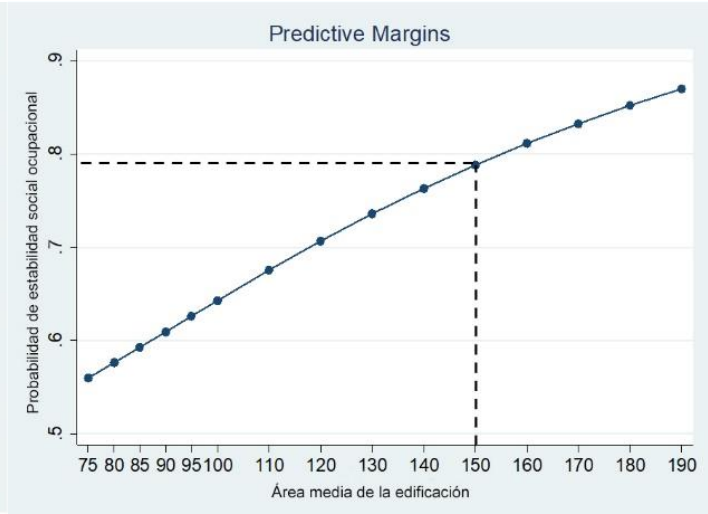

(b)

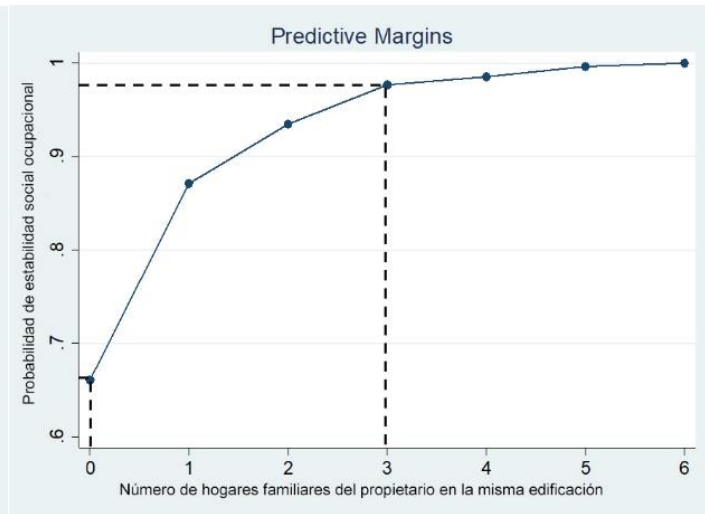

(d)

Figura 1 - Análisis marginal modelo inmovilidad laboral ocupacional. (a) Cambios de trabajo; (b) Tamaño de la edificación en metros cuadrados; (c) Número de hogares, (d) Red familiar en la vivienda. Fuente: Cálculos de los autores.

Una vivienda de mayor tamaño inmoviliza ocupacionalmente al individuo. Un propietario de una edificación de $75 \mathrm{~m}^{2}$ tiene una probabilidad de $56 \%$ de ser estable ocupacional (Figura 1), mientras que con una vivienda de $150 \mathrm{~m}^{2}$ del $76,3 \%$. Un número mayor de hogares reduce la inmovilidad con $90 \%$ de significancia estadística. El incremento del número de hogares en la vivienda reduce la probabilidad de inmovilidad. Si la vivienda tiene solo un hogar, la probabilidad de inmovilidad ocupacional es de 89\%, pero si se incrementa a 3 hogares, se reduce al 59\%. Sin embargo, la red familiar aumenta la probabilidad de inmovilidad. Cuando hay más hogares familiares que comparten la edificación con el propietario la probabilidad de inmovilidad es mayor. Tener 3 hogares con vínculo familiar asegura la inmovilidad ocupacional del propietario.

Nótese que la construcción de una edificación grande deja al individuo con un objetivo de vida principal que aumenta su inmovilidad laboral. Una vez construida, puede arrendar a más hogares en lo que no solo le genera mayores ingresos y le da algo de libertad ocupacional, se podría arriesgar a otras ocupaciones. Al evaluar las diferencias en las probabilidades de duplicar el tamaño de la casa 20.3 frente a pasar de 1 a 3 hogares, 30 puntos porcentuales, en el agregado el tamaño importa sí y solo sí no tiene familiares en la vivienda. 
Si bien las diferencias educativas son bajas en esta población de estudio, y de allí la no significancia estadística, en los marginales se aprecia lo positivo de la mayor educación como factor clave para promover la movilidad social. El propietario sin educación tiene una probabilidad del $75,6 \%$ de ser inmovil ocupacionalmente, el técnico o tecnólogo un $56 \%$ de probabilidad. Aquellos que dijeron tener secundaria tienen un $90 \%$ de probabilidad de ser inmóvil y los profesionales no están en esta condición de inmovilidad ocupacional.

Solo los individuos pertenecientes al grupo de ingresos entre 300 y 600 mil pesos ${ }^{6}$ son estadísticamente significativos. Tienen una probabilidad de $96 \%$ de ser inmóviles. En los marginales la probabilidad de ser inmóviles se reduce con el incremento del ingreso. En el grupo de ingresos de 1,2 y 2,4 millones $^{7}$ la probabilidad es del 71\%. Un mayor nivel de ingresos reduce la probabilidad de ser inmóvil. Respecto de los aspectos para mejorar en la vida tener un empleo formal y estable como el aspecto más importante para mejorar económicamente, se asocia con menor probabilidad de estar en condición de inmovilidad ocupacional. La idea de tener una casa grande como el aspecto más importante para mejorar en la vida se asocia con la mayor probabilidad (93\%) de ser inmóviles. El estudio, trabajar duro y la fe en Dios no son estadísticamente significativas.

En conclusión, lo interesante son los resultados netos de las variables en el largo plazo. Una casa grande con varios hogares no familiares aumenta el ingreso que permitiría una apuesta por un mayor nivel educativo y tener más cambios de trabajo que incrementan la probabilidad de movilidad ocupacional. Estos resultados son consistentes con las variables de percepción. Quienes apuestan por la educación y el trabajo como aspectos para mejorar en la vida tienen una menor probabilidad de ser inmóviles, el problema es su financiamiento de corto plazo. Como los ingresos aparecen como una variable importante en el análisis de la movilidad ocupacional, se exploró en el siguiente modelo.

\section{Sobre las trayectorias residenciales de los propietarios}

En este subcapítulo se avanzará en un análisis de las trayectorias residenciales de los propietarios a lo largo de su vida hasta la llegada a la vivienda actual. La distribución de la intensidad de la movilidad residencial (número de cambios de residencia) no es gaussiana de manera que se trabaja con la moda y la frecuencia acumulada de las primeras tres etapas. En general, los propietarios tienen una moda de 3 cambios residenciales. Como se aprecia en la Tabla 6, el 70,89\% de los propietarios de estos barrios tienen máximo tres etapas. En el barrio San Martín de Porres los propietarios han tenido la menor movilidad residencial, el 84,62\% han cambiado como máximo tres veces de residencia. Por el contrario, en el barrio El Anhelo solo el 55,84\% de los propietarios tienen la condición anterior. En Perdomo y Japón la intensidad de la movilidad es un poco más baja que el promedio y en Atenas se iguala a la situación general.

Tabla 6 - Frecuencia acumulada de las primeras 3 etapas

\begin{tabular}{lll}
\hline Número de Etapas & Frecuencia & Acumulado \\
\hline Total & 224 & 70,89 \\
Atenas & 35 & 70 \\
El Anhelo & 43 & 55,84 \\
Japón & 37 & 72,55 \\
Perdomo & 65 & 75,58 \\
San Martin de Porres & 44 & 84,62 \\
\hline
\end{tabular}

Fuente: Cálculos de los autores.

\footnotetext{
6 Entre 85 y 170 dólares aproximadamente.
}

7 Entre 340 y 680 dólares aproximadamente. 
El porcentaje de propietarios que no se han movido nunca o solo han tenido un cambio de residencia, es de 54,9\% en el San Martín de Porres. La inmovilidad residencial en sentido estricto, es decir quienes han vivido siempre en la misma vivienda, se presenta en el $26 \%$ de los casos en el barrio San Martín de Porres, en Atenas el 5 \% y en el Perdomo solo el 1,3 \%. En Japón y El Anhelo, no hay ningún caso de este tipo. De acuerdo con estos datos, la intensidad de la movilidad varía con el barrio, en lo que puede influir su historia, su antigüedad e incluso su localización. Los propietarios de los barrios más antiguos se movieron menos veces antes de llegar a su vivienda actual, lo que insinúa un cambio en las dinámicas de la inserción territorial.

En cuanto a la duración de las etapas residenciales, se encuentra que tienden a acortarse con el tiempo. La primera movilidad suele ser de independencia, o en otros casos de movilidad junto con los padres, generalmente migración. La última movilidad es la llegada a la vivienda actual, que suele ser el paso de arrendatario a propietario. En la Tabla 7 se presentan las estimaciones de una regresión lineal de la duración de la primera etapa como función del año de inicio y las predicciones en los años 1940 y 2005.

Tabla 7 - Duración de la primera etapa y Predicciones

\begin{tabular}{lll}
\hline DuraciónE1 & Estimador & t-Student \\
\hline Año de inicio & -.1793024 & -4.40 \\
Constante & 369.2425 & 4.63 \\
\hline Linear prediction & & \\
\hline 1940 & 21.39583 & 17.72 \\
2005 & 9.741172 & 6.60 \\
\hline
\end{tabular}

Fuente: Cálculos de los autores.

En la tabla el coeficiente del año de inicio indica que por cada año en que se aproxima al tiempo actual, la duración de la etapa se reduce en 0.179 años. Si la primera etapa inició en 1940 su duración media es de 21.4 años, mientras que si inició en 2005 se reduce a 9.74 años. Ahora, como la media del año de inicio es 1960, su duración es de 17.8 años lo que sugiere la independencia residencial. Para cada etapa se estableció si se trataba de migración a Bogotá u otras ciudades. Las movilidades intraurbanas se clasificaron según fuera entre localidades, intra localidad, intra barrio o en la misma vivienda. Las movilidades tienden a acortarse también en distancia, la primera movilidad es principalmente migratoria y las últimas intralocales e intrabarriales, lo que refleja los procesos de poblamiento de la ciudad (Dureau et al., 2015).

Solo el 12,4\% de los encuestados llegó a la vivienda actual directamente desde otra ciudad o municipio, el 41,5\% venían de otra localidad de la ciudad, el 12,4\% de los casos vivían antes en otro barrio de la misma localidad, el 20,3\% en el mismo barrio y el $13.4 \%$ se movieron en la misma casa. Se encontraron diferencias entre los barrios, así, la mayor parte de las movilidades hacia la vivienda actual en San Martín de Porres (90,9\%) y Atenas (65,8\%), fueron cercanos (misma localidad, barrio o edificación). En El Anhelo y en Japón $(76,5 \%)$ son mucho más importantes las movilidades desde otras localidades de la ciudad, muy pocos propietarios se han movido previamente en el mismo barrio. Tanto en Perdomo como en Atenas los movimientos en la misma edificación se acercan al 23\%, mucho mayores que en los demás barrios analizados.

La gran mayoría de movilidades se dan hacia una casa $(79,2 \%)$ y vivian en una casa antes de vivir en la actual vivienda (71,4\%), el 15,4 \% vivía en un cuarto y el 9,9 en un apartamento. Con respecto a la tenencia, la mayoría pasa de arrendatario a propietario $(27,2 \%)$. El siguiente cambio en importancia es la continuidad como arrendatario (20,8\%). El 45\% vivía en arriendo antes de mudarse a la vivienda actual y el $25.3 \%$ en la vivienda de otro miembro del hogar.

\section{Variables asociadas a la movilidad residencial}

Con el fin de indagar por variables explicativas de la intensidad de la movilidad residencial se realizó un modelo de regresión lineal clásico en donde el número de etapas residenciales se explica por las variaciones en los cambios de trabajo, la edad del propietario, el género, la tenencia de la primera etapa residencial, el logaritmo del área del terreno, el número de hogares por vivienda, la red familiar (hogares familiares en la 
vivienda), el número de hijos, el año de adquisición de la propiedad, el año de mudanza y la movilidad social. En la Tabla 8 la primera columna corresponde a las variables explicativas, la segunda a sus estimadores y la tercera el estadístico de prueba de significancia estadística de una regresión con errores robustos.

Tabla 8 - Estimación del Número de etapas Residenciales

\begin{tabular}{llc}
\hline Número de etapas & Estimador & t-student \\
\hline Cambios de trabajo &, 2571605 & $2,05^{*}$ \\
Edad del propietario &, 0166445 & $2,47^{* *}$ \\
Género &,- 1067781 & $-0,65^{*}$ \\
Tenencia primera etapa & & $-1,28$ \\
$\quad$ De propietario a arrendatario &,- 5154182 & $-3,51^{*}$ \\
De vivienda familiar a propietario & $-1,534837$ & 0,49 \\
De vivienda familiar a vivienda familiar &, 2275971 & $-0,39$ \\
De vivienda familiar a arrendatario &,- 1580026 & $-3,28^{*}$ \\
De arrendatario a propietario & $-1,460779$ & $-0,98$ \\
De arrendatario a vivienda familiar &,- 580289 & 0,21 \\
De arrendatario a otra forma de tenencia &, 0951861 & $-2,40$ \\
Logaritmo Área del terreno &,- 3601458 & 1,50 \\
Hogares por vivienda &, 0876307 & 0,36 \\
Red familiar &, 0413148 & 1,55 \\
No de hijos &, 1023177 & 2,43 \\
Año adquisición de la propiedad &, 0220713 & $-0,29$ \\
Año de mudanza &,- 0027173 & \\
Movilidad Social Ocupacional & & 0,15 \\
Descendente &, 0395079 & $-0,61$ \\
Ascendente &,- 1368286 & $-2,63$ \\
Constante & $-34,81462$ & 0,4695 \\
R-squared & & \\
\hline
\end{tabular}

Fuente: Cálculo de los autores.

Si bien los resultados tienen interpretación directa, se describen junto con los efectos marginales por la magnitud de algunos estimadores. De acuerdo con la estimación, un cambio de trabajo adicional incrementa en 0,257 etapas la movilidad residencial. Pasar de cero a 5 trabajos aumenta en 1.29 las etapas residenciales (Tabla 9). Si la edad del propietario se incrementa en un año el número de etapas lo hace en 0.016 años. El diferencial marginal entre 40 y 60 años es de 0,33 cambios residenciales a favor del último. El género no marca diferencias en los cambios residenciales (Tabla 9).

La tenencia en la primera etapa de movilidad residencial reduce de manera significativa la movilidad residencial. El traslado de una vivienda familiar a una vivienda propia reduce 1,53 etapas frente al paso de una vivienda propia a una vivienda de otro miembro del hogar. El paso de arrendatario a propietario reduce en 1,46 el número de etapas residenciales. Es decir, la propiedad inmoviliza a los propietarios.

Tabla 9 - Efectos marginales

\begin{tabular}{cl}
\hline Variable & Margins \\
\hline Cambios de trabajo & $2.598866^{*}$ \\
0 & $3.884669^{*}$ \\
5 & \\
Edad del propietario & $2.974513^{*}$ \\
40 & $3.307402^{*}$ \\
60 & \\
Tenencia en la primera etapa & $3.708^{*}$ \\
De propietario a vivienda familiar & $2.173163^{*}$ \\
De vivienda familiar a propietario & $2.247221^{*}$ \\
De arrendatario a propietario & \\
Año de adquisición de la casa & $2.643698^{*}$ \\
1960 & $3.636905^{*}$ \\
2005 &
\end{tabular}

Fuente: Cálculos de los autores. 
La elasticidad del área del terreno indica que duplicar la moda de 72 metros cuadrados se traduce en una reducción de la movilidad residencial en 1 etapa. Una mayor área de terreno implica mayor esfuerzo en la construcción de la vivienda y mayor ingreso futuro de los arriendos. El efecto marginal de la edad de adquisición de la propiedad es una etapa adicional cuando la adquisición fue en 2005 frente a 1960. Las demás variables no tienen significancia estadística.

\section{Reflexiones sobre los hallazgos para concluir}

Las trayectorias laborales y residenciales de los propietarios en los sectores populares estudiados se caracterizan por la inmovilidad. Muy pocos han logrado un ascenso ocupacional a lo largo de su vida. Si bien no es el interés central del artículo, es importante mencionar que hay cuestiones estructurales asociadas a los procesos de movilidad e inmovilidad social de los individuos y los hogares en los sectores populares en América Latina, asociados a la estructura de clases sociales y a las características propias de los procesos de urbanización de la región (Jaramillo, 2008). Más recientemente la implementación de políticas urbanas de corte neoliberal han consolidado una aguda y persistente desigualdad del ingreso y unas ciudades profundamente fragmentadas y polarizadas en las que persiste la existencia de periferias urbanas y metropolitanas en condiciones de alta precariedad (Pradilla \& Márquez, 2008). Esto para remarcar que si bien este texto profundiza en aspectos propios de los hogares, las viviendas y las características de los individuos, estos operan en el marco de constreñimientos sociales y de las estructuras de oportunidad existentes.

Ahora bien, los datos de este estudio confirman que aspectos como el bajo nivel educativo, pocos cambios de trabajo, bajos ingresos, una vivienda grande y albergar varios hogares con vínculo familiar explican una parte importante de la inmovilidad ocupacional en los sectores populares. Estas variables están estrechamente vinculadas, con un bajo capital educativo, los cambios de trabajo a otras actividades apenas movilizan ocupacionalmente a algunos de ellos. La vivienda se aprecia como un proyecto de vida intergeneracional, los hoy viejos trabajaron para el sostén futuro no solo de sí mismos, sino de su familia extendida. En este sentido, el tamaño de las viviendas de hoy confirma esta apreciación.

La migración a Bogotá, a vivienda en arriendo en zonas centrales de la ciudad antecede la llegada a la vivienda definitiva, a pesar de las diferencias en las trayectorias residenciales entre barrios. Las primeras etapas suelen ser de mayor duración temporal que las finales y estas últimas de menor distancia, dentro del barrio, aun dentro de la misma casa. Con la evidencia recogida, las trayectorias residenciales y ocupacionales no están inter-relacionadas. Si bien la mayoría son inmóviles laboral y residencialmente, cuando se mueven física y ocupacionalmente, su trayectoria es de corto alcance.

Como lo mencionaban Dureau et al. (2015), "la movilidad tiene un rol decisivo en la construcción de las desigualdades" (p. 342) así como también en su reproducción (Tilly, 2008; Bourdieu, 1989). La inmovilidad residencial y social, evidente en estos sectores, son fenómenos estrechamente relacionados. En este estudio solo logró comprobarse una simultaneidad de estas inmovilidades, pero otros, como el de Perlman (2010) ya habían sugerido una relación causal, atribuida a la propiedad de la vivienda asociada a una mayor inmovilidad social y residencial.

A pesar de ser la propiedad de la vivienda un sueño cumplido de los entrevistados y un símbolo de su movilidad social, los resultados indican que a largo plazo incrementa la inmovilidad residencial, y en muchos casos los recursos económicos y humanos dedicados a ella van en detrimento de la acumulación de capital humano (Moser, 2009). No se recogió información sobre los mecanismos que configuran las decisiones de inmovilidad residencial, de hecho, en la encuesta se preguntó por el interés de mudarse, a lo que la mayoría (80\%) respondió negativamente. Lo cierto es que aquellos que querían hacerlo lo encontraban muy dificil de concretar, estos hallazgos confirman las dificultades de movilizar estas viviendas en el mercado (Ward \& Otros, 2015). Por otra parte, la expansión de una cultura de la propiedad en los países tiende a ser inversamente proporcional a la fortaleza de los sistemas de seguridad social. Para la 
población de bajos ingresos, la propiedad es un elemento de seguridad ante la incertidumbre del sistema capitalista (Gilbert, 2001).

Los resultados netos del modelo en el largo plazo sugieren que una casa grande aumenta el ingreso que permitiría una apuesta por un mayor nivel educativo en las siguientes generaciones. No se necesita terminar la casa, por el camino se va arrendando, de manera que esto genera algo de movilidad al propietario y libera recursos para formación de capital humano. En consecuencia, las redes familiares en la vivienda disminuyen la movilidad ocupacional, dado que limita la posibilidad de obtención de rentas adicionales ${ }^{8}$.

Los altos precios del suelo y la producción industrial de vivienda limitan la autoconstrucción de la casa como mecanismo individual de ascenso. Al ser la educación un canal de movilidad social y una exigencia en el mercado laboral actual, define el rumbo de la movilidad social y residencial contemporánea.

\section{Referencias}

Abramo, P. (2008). El mercado del suelo informal en favelas y la movilidad residencial de los pobres en las grandes metrópolis: un objeto de estudio para América Latina. Territorios, (18-19), 55-73. Recuperado en 03 de mayo de 2021, de https://revistas.urosario.edu.co/index.php/territorios/article/view/827

Álvarez, M. J. (2007). Asentamientos irregulares montevideanos: la desafiliación resistida. Cadernos Metrópole, (18), 207249. Recuperado en 03 de mayo de 2021, de https://www.redalyc.org/articulo.oa?id=402837797010

Authier, J. , Bidet, J., Collet, A., Gillbert, P., \& Steinmetz, H. (2010). Etat des lieux sur les trajectoires résidentielles. Paris: Ministère de l'Ecologie, de l'Energie, du Développement Durable et de la Mer DGALN/Plan Urbanisme Construction Architecture.

Bonvalet C., \& Dureau, F. (2000). Los modos de habitar: una decisión condicionada. En Dureau F., Dupont. V.; Lelièvre, É.; Lévy, J.P. y Lulle, T. (Coords.) Metrópolis en Movimiento. Una Comparación Internacional (pp. 69-87) Bogotá: Alfaomega IRD - Universidad Externado de Colombia.

Bourdieu, P. (1989). La ilusión biográfica. Historia y Fuente Oral, (2), 27-33. Recuperado en 03 de mayo de 2021, de http://www.jstor.org/stable/27753247

Brown, L., \& Moore, E. (1970). The Intra-Urban Migration Process: A Perspective. Geografiska Annaler, 52(1), 368-381. http://dx.doi.org/10.1080/04353684.1970.11879340

Camargo, A. (2020). Vivienda y estrategias familiares de vida en barrios populares consolidados en Bogotá. Revista INVI, 35(98), 101-125. Disponible en: http://revistainvi.uchile.cl/index.php/INVI/article/view/1500

Contreras, Y. (2012). Cambios socio-espaciales en el centro de Santiago de Chile: Formas de anclarse y prácticas urbanas de los nuevos habitantes (tesis de doctorado). Doctorado en Geografía, Universidad de Poitiers, Poitier, Francia.

Delaunay, D., \& Dureau, F. (2004). Componentes sociales y espaciales de la movilidad residencial en Bogotá. Estudios demográficos y urbanos, (55), 77-113. http://dx.doi.org/10.24201/edu.v19i1.1196

Di Virgilio, M. M. (2011). La movilidad residencial: una preocupación sociológica. Territorios, (25), 173-190. Disponible en: https://revistas.urosario.edu.co/index.php/territorios/article/view/1851

Dieleman, F. (2001). Modelling residential mobility; a review of recent trends in research. Journal of Housing and the Built Environment, 16, 249-265. https://doi.org/10.1023/A:1012515709292

Duhau, E. (2003). División social del espacio y movilidad residencial. Papeles de población, 9(36), 161-21. Recuperado en 03 de mayo de 2021, de http://www.scielo.org.mx/scielo.php?script=sci_arttext\&pid=S1405-

$74252003000200008 \& \operatorname{lng}=$ es\&tlng=es.

Dureau F., Lulle, T., Souchaud, S., \& Contreras, Y. (Eds.). (2015). Movilidades y cambio urbano. Bogotá, Santiago y San Pablo. Bogotá: Universidad Externado de Colombia.

Dureau, F. (2010). Vivir en Bogotá: estrategias y prácticas de movilidad residencial urbana. En: Jaramillo, S. (Ed.). Bogotá en el cambio de siglo: promesas y realidades (pp. 53-86). Quito: OLACHI.

8 Una mirada sobre la obtención de ingresos mediante la vivienda como parte de las estrategias de vida revisar Camargo (2020). 


\section{Movilidad residencial y social en barrios populares consolidados en Bogotá}

Erikson, R., \& Goldthorpe, J. (1992). The Constant Flux: a Study of Class Mobility in Industrial Societies. Oxford: Oxford University Press.

Gilbert, A. (2001). ¿Una casa es para siempre? Movilidad Residencial y Propiedad de la Vivienda en Asentamientos Autoproducidos. Revista Territorios, Julio, 006, 51-63. Recuperado en 03 de mayo de 2021, de https://revistas.urosario.edu.co/index.php/territorios/article/view/5652

Jaramillo, S. (2008). Reflexiones de la "informalidad" fundiaria como peculiaridad de los mercados de suelo en América Latina. Territorios, (18-19), 11-53. Recuperado en 03 de mayo de 2021, de https://revistas.urosario.edu.co/index.php/territorios/article/view/826

Kessler, G., \& Espinoza, V. (2003). Movilidad social y trayectorias ocupacionales en Argentina: rupturas y algunas paradojas del caso de Buenos Aires. Chile: Cepal. Recuperado en 03 de mayo de 2021, de https://repositorio.cepal.org/handle/11362/6038

Knox, P. (1982). Urban Social Geography: an Introduction. Londres: Longman.

Leal, J. (2002). Segregación social y mercados de vivienda en las grandes ciudades. RES. Revista Española de Sociología, (2), 59-75. Recuperado en 03 de mayo de 2021, de https://recyt.fecyt.es/index.php/res/article/view/64866

Lulle, T. (1998). Prácticas y Representaciones en torno a la vivienda y a la ciudad: el caso de los sectores medios en Bogotá. En Campos, Y. y Ortiz, I. (Comp.) La Ciudad Observada: Violencia, Cultura y Política (pp. 463-490). Alcaldía Mayor de Bogotá.

Menna, S. (2000). São Paulo: el acceso a la propiedad a cualquier precio. En: Françoise D.; Dupont. V.; Lelièvre, É.; Lévy, J.P. y Lulle, T. (Coords.). Metrópolis en movimiento. Una Comparación Internacional. (139-146) Bogotá: Alfaomega - IRD Universidad Externado de Colombia.

Módenes, J. (2008). Movilidad espacial, habitantes y lugares: retos conceptuales y metodológicos para la geodemografía. Estudios Geográficos, LXIX, 264, 157-178. https://doi.org/10.3989/egeogr.2008.i264.83

Moser, C. (2009). Gente del barrio, vidas extraordinarias. Activos y reducción de la pobreza en Guayaquil 1978-2004. Santiago de Chile: Ediciones Sur.

Padilla, E. (2012). Movilidad residencial en asentamientos de origen irregular en guadalajara 1970 - 2009. (Doctorado) Doctorado en Ciudad, Territorio y Sustentabilidad, Universidad de Guadalajara, Guadalajara, México.

Palomares-Linares, I., \& Van Ham, M. Del sedentarismo a la hipermovilidad. Medida y determinantes de las historias de (in)movilidad residencial en contextos urbanos. Papers. Revista de Sociologia, [S.l.], 102(4), 637-671. Recuperado en 03 de mayo de 2021, de https://dialnet.unirioja.es/servlet/articulo?codigo=6153219

Perlman, J. (2010). Favela. Four decades of living on the edge in Rio de Janeiro. Oxford: Oxford University Press.

Pujadas, I. (2009). Movilidad residencial y expansión urbana en la región metropolitana de Barcelona, 1982-2005. Revista electrónica de geografía y ciencias sociales, XIII (290). Recuperado en 03 de mayo de 2021, de http://www.ub.edu/geocrit/sn/sn-290.htm

Pradilla, E., \& Márquez, L. (2008). Presente y futuro de las metrópolis en América Latina. Territorios, 18-19, $147-181$.

Rodríguez, J. (2007). Segregación residencial, migración y movilidad espacial. El caso de Santiago de Chile. Cadernos metrópole, 17, 135-168.

Rossi, P. (1955) Why Families Move. Sage Publications, 2 nd edición (1 marzo 1980)

Servicio Nacional de Aprendizaje SENA, \& Ministerio del Trabajo de Colombia (2015). Clasificación Nacional de Ocupaciones 2015. Ministerio del Trabajo de Colombia. Recuperado en 03, 05, 2121, de https://observatorio.sena.edu.co/Content/pdf/cno2015.pdf

Secretaría Distrital de Planeación (2015) Resultados de la Encuesta Multipropósito 2014. Alcaldía Mayor de Bogotá. Recuperado en 03 de mayo de 2021, de http://www.sdp.gov.co/sites/default/files/1._boletin_resultados_encuesta_multiproposito_2014.pdf

Secretaría Distrital de Planeación (2018) Barrios legalizados en Bogotá. Alcaldía Mayor de Bogotá. Recuperado en 03, 05, 202103 de mayo de 2021, de http://www.sdp.gov.co/transparencia/informacion-interes/datos-abiertos/barriolegalizado-bogota-dc

Sorokin, P. (1961). Estratificación y Movilidad Social. Revista Mexicana De Sociología, 15(1), 83-117.

http://dx.doi.org/10.2307/3537850 


\section{Movilidad residencial y social en barrios populares consolidados en Bogotá}

Stake, R (1994). Case Studies. En: Norman K. D. y Yvonna L. (eds.) Handbook of Qualitative Research, Thousand Oaks (pp. 236-245). Sage Publication.

Suarez, M., \& Delgado, J. (2010). Patrones de movilidad residencial como evidencia de la co-localización de población y empleos. Eure, 36(107), 67-91.

Tilly, C. (2008). La pobreza y la política de exclusión. En: Nayaran, D., \& Petesch, P. (Coords.). Salir de la pobreza: perspectivas interdisciplinarias sobre la movilidad social (pp. 47-76). Banco Mundial.

Torche, F., \& Worlmand, G. (2004). Estratificación y Movilidad Social en Chile: Entre la adscripción y el logro. Santiago de Chile: Cepal.

Uribe, C., \& Pardo, C. (2006). La ciudad vivida: movilidad espacial y representaciones sobre la estratificación social en Bogotá. Universitas humanística, (62), 169-203.

Valenzuela, E., \& Herrera, S. (2003). Movilidad residencial y movilidad social. En: INE (Ed.), Cuanto y Como Cambiamos los Chilenos. Balance de una Década (pp. 105-133). Santiago de Chile: Instituto Nacional de Estadística.

Ward, P. (2009). Hacia una segunda etapa de la regularización de los títulos de propiedad en México y Colombia. Papeles de Coyuntura. 27. Recuperado en 03 de mayo de 2021, de http://repository.unipiloto.edu.co/bitstream/handle/20.500.12277/8419/Segunda_etapaPapelesCoyuntura27.pdf?sequence=1\&isAllowed $=y$

Ward P.M.; Jiménez, E.; Di Virgilio, M y Camargo, A. (2015). Políticas de Vivienda en Ciudades Latinoamericanas: una nueva generación de estrategias y enfoques para 2016 ONU-Habitat III. Editorial Universidad del Rosario.

Zamorano, C. (2007). Los hijos de la modernidad: Movilidad social, vivienda y producción del espacio en la Ciudad de México. Alteridades, 17(34), 75-91.

Editor: Paulo Nascimento Neto

Recibido: 3 abr. 2020

Aprobado: 15 dic. 2020 